\title{
La salud bucal \\ como derecho humano y bien ético
}

\section{Oral health as a \\ human right and ethical good}

\section{A saúde bucal como direito humano e bem ético}

Fecha de recepción: 20 de septiembre de 2016

Fecha de evaluación: 15 de noviembre de 2016

Fecha de aceptación: 30 de noviembre de 2016

Disponible en línea: 7 de diciembre de 2016

\author{
Gabriela Rueda Martínez* \\ Aline Albuquerque ${ }^{* *}$ \\ DoI: http://dx.doi.org/10.18359/rlbi.2299.
}

Cómo citar:

Rueda Martínez, G. y Albuquerque, A. (2016). La salud bucal como derecho humano y bien ético. Revista Latinoamericana de Bioética, 17(1), 36-59. DoI: http://dx.doi.org/10.18359/rlbi.2299

\footnotetext{
* Artículo de investigación.

* Odontóloga, Universidad Santo Tomás. Especialista y Magister en Bioética, Programa de Pós-Graduaçao em Bioética, Universidad de Brasília. Estudiante de la Doctorado de la Universidad de Brasília. Correo electrónico institucional: gabrielarueda@aluno.unb.br. ORCID: http://orcid. org/0000-0001-7135-4293. Bogotá, Colombia.

* Abogada, Post-Doctora en Derechos Humanos e investigadora visitante del Centro de Derechos Humanos de la Universidad de Essex, Inglaterra- Doctora en Ciencias de la Salud, área de concentración en Bioética, Universidad de Brasilia y Universidad de Zurich. Profesora del Programa de Pós-Graduaçao em Bioética de la Universidad de Brasília. Correo electrónico institucional: aline.santanna@sdh.gov.br. ORCID: http://orcid.org/0000-00025568-0790. Brasília/DF, Brasil.
} 


\title{
Resumen
}

Fue ejecutado un examen del Reporte Mundial de Salud Bucal, adoptado por el Programa de Salud Bucal Global de la Organización Mundial de la Salud, con el objetivo de determinar si este documento se constituye en una herramienta teórico-política para alcanzar el derecho a la salud bucal. La metodología empleada fue el análisis de prácticas discursivas como producción del sentido. El análisis constató que el Reporte Mundial de Salud Bucal no se constituye en una herramienta política-teórica para la satisfacción del derecho a la salud bucal, en razón a que no incorpora el enfoque basado en los derechos humanos. Así pues, la perspectiva de este documento es limitada a los cuidados en salud bucal y no establece mecanismos para el efectivo enfrentamiento de los determinantes sociales y las obligaciones estatales de asegurar el derecho a la salud bucal no son enfatizadas. Asimismo, al alejarse del referencial de los derechos humanos, también se distancia de la bioética, en razón de que el paciente no es considerado en sus múltiples contextos, que permean el proceso salud-enfermedad; así, es contrario a la promoción de condiciones de vidas justas y ecuánimes para los individuos.

Palabras clave: bioética, derechos humanos, derecho a la salud, odontología.

\begin{abstract}
An examination of the World Oral Health Report, adopted by the Global Oral Health Program of the World Health Organization, was executed out to determine if this document constitutes a theoretical-political tool to achieve the realization of the right to Oral health. The applied methodology was the analysis of discursive practices as production of the sense. The analysis found that the World Oral Health Report does not constitute a political-theoretical instrument for the satisfaction of the right to oral health because it does not incorporate the human rights approach. Thereby, the perspective of this document is limited to oral health care and does not establish mechanisms for the effective confrontation of social determinants and the state's obligations to ensure the right to oral health are not emphasized. Also, moving away from the referential of human rights, it also distances itself from bioethics, because the patient is not considered in its multiple contexts, which permeate the health-disease process; thus, it is contrary to the promotion of conditions of just and equal lives for individuals.
\end{abstract}

Keywords: bioethics, human rights, right to health, dentistry.

\section{Resumo}

Foi executado um exame do Relatório Mundial da Saúde Bucal, adotado pelo Programa de Saúde Bucal Global da Organização Mundial da Saúde, com o objetivo de determinar se este documento constitui-se numa ferramenta teórica-política para alcançar a realização do direito à saúde bucal. A metodologia utilizada foi a análise de práticas discursivas como produção do sentido. A análise constatou que o Relatório Mundial de Saúde Bucal não se constitui numa ferramenta teórica-política para a satisfação do direito à saúde bucal, porque não incorpora a abordagem baseada nos direitos humanos. Assim então, a perspectiva deste documento é limitada aos cuidados em saúde bucal e não estabelece mecanismos de enfrentamento eficaz dos determinantes sociais e as obrigações do Estado de garantir o direito à saúde bucal não são enfatizadas. Além disso, ao ficar longe do referencial dos direitos humanos, também toma distância da bioética, em ração de que o paciente não é considerado nos seus múltiplos contextos, que permeiam o processo saúde-doença; assim, é contrário à promoção de condições de vidas justas e imparciais para os indivíduos.

Palavras-chave: bioética, direitos humanos, direito à saúde, odontologia. 


\section{Introducción}

El presente artículo está orientado a analizar el referencial teórico-normativo del derecho a la salud y direccionarlo al análisis de documentos promulgados por agencias internacionales en salud, aplicados al campo de la salud bucal global (Petersen, 2003).

La satisfacción del derecho a la salud es un tópico de la bioética y es necesaria para alcanzar la sobrevivencia humana basada en la dignidad (Potter y Potter, 1995); este parámetro axiológico ha sido reconocido como núcleo esencial compartido entre la bioética y los derechos humanos; además, estos campos del conocimiento objetivan delimitar determinadas prácticas sociales y proteger los bienes éticos, ${ }^{1}$ entre los cuales se destaca la salud (Albuquerque, 2010).

El derecho a la salud desde la perspectiva de la bioética reconoce que los conflictos

Definidos como aspectos concretos del bienestar de los seres humanos, vinculados a la vida y a las condiciones que la hacen digna, cuyo alcance está determinado por la cooperación entre las personas. Estos bienes están contenidos en los derechos humanos y poseen naturaleza colectiva y carácter inviolable (Albuquerque, 2010). La alusión a los bienes éticos en instrumentos normativos internacionales de bioética se manifiesta en la Declaración universal sobre Bioética y derechos Humanos (dubdh), especialmente en su Artículo 14, que trata sobre la responsabilidad social y la salud, caracterizándola como un "bien social y humano" (p. 7) (Organização das Nações Unidas para a Educação, a Ciência e a Cultura Unesco, 2005). éticos derivados de las prácticas clínicas no se originan únicamente de las fragilidades de la relación profesional-paciente, sino que son consecuencia de otros hechos sociales relacionados con los determinantes sociales de la salud, a las políticas públicas, a la gestión institucional y a la distribución de los recursos sanitarios por parte del Estado. En este sentido, el reconocimiento de este derecho como tema de la bioética se fundamenta en la visión del paciente como un ser holístico, sujeto de derechos, incorporado en diversidad de contextos.

El propósito de este artículo es determinar si el Reporte Mundial de Salud Bucal de la oms se constituye en una herramienta teórico-política para alcanzar la realización del derecho a la salud bucal. Para esto, fueron formuladas dos partes: la primera expone las bases conceptuales del derecho a la salud, abordando la Observación 14 de 2000, proclamada por el Comité de Derechos Económicos, Sociales y Culturales (CDESC) (United Nations Economic and Social Council, 2000). También en la primera es presentado el fundamento conceptual de la salud bucal, investigado a partir de búsqueda bibliográfica en las siguientes bases de datos: Google Scholar, PubMed, MedLine y Scielo, a través de los siguientes descriptores; "oral health and human rights"; "oral health and bioethics"; "right to oral health", "oral health and quality of life", empleados en español y portugués.

La segunda parte expone el análisis del Reporte Mundial de Salud Bucal, 
presentado en la siguiente subdivisión: explicación del abordaje metodológico, presentación del documento en examen, exposición de los resultados y la discusión, en un único punto y conclusiones. Fue adoptada la metodología propuesta por Spink y Giménez (1994) sobre análisis de prácticas discursivas, cuyo enfoque es el lenguaje utilizado, en este caso concreto, aplicado a un documento de dominio público. El Reporte Mundial de Salud Bucal se constituye en prácticas discursivas producidas por una autoridad internacional en salud bucal, dirigida a los Estados parte; por tanto, el examen de sus repertorios lingüísticos revelará la postura de esta agencia de las Naciones Unidas sobre el derecho a la salud bucal y la incorporación de las prescripciones del referencial del derecho a la salud. A continuación, serán presentados los pilares que fundamentan el análisis.

\section{Fundamentación teórica}

El derecho a la salud en la Observación 14 de 2000. El presente artículo enfatiza la Observación 14 de 2000, reconocida como fundamento para la comprensión y aplicación concreta del artículo 12 del Pacto Internacional de Derechos Económicos, Sociales y Culturales, que trata sobre el derecho a la salud (United Nations, General Assembly, 1966).

La Observación 14 se divide en cinco partes: contenido normativo, ${ }^{2}$ obliga-

2 El contenido normativo está previsto concretamente en el artículo 12.1 de la Observación 14, que incorpora la definición del derecho a la salud y en ella, sus determinantes sociales; ciones de los Estados parte, violaciones del derecho a la salud, aplicación en el plano internacional y obligaciones de los actores diferentes a los Estados parte (United Nations Economic and Social Council, 2000).

Considerando esta estructura, el marco de referencia que direccionó el análisis del presente estudio fue subdividido en tres ejes: contenido del derecho a la salud, que incluye sus determinantes sociales, elementos de este derecho y obligaciones estatales. A continuación, serán descritos cada uno de estos puntos.

\section{Conceptuación del derecho a la salud}

El derecho a la salud es dependiente e indispensable para el ejercicio de otros derechos humanos; por esta razón, todo ser humano es titular del derecho de disfrutar el más alto nivel posible de salud, ${ }^{3}$ que conduce a vivir una vida digna.

La salud es un derecho inclusivo, es decir, va más allá del derecho a la atención sanitaria oportuna y apropiada, e incorpora el derecho a los determinantes sociales de la salud. Entre ellos se encuentran el acceso a agua limpia y potable, condiciones sanitarias adecuadas, suministro de nutrición apropiada, buenas condiciones de residencia, condiciones sanas de trabajo y acceso a educación

este artículo también abarca sus elementos constitutivos.

3 Denominación alterna al Derecho a la Salud; en el presente artículo, serán utilizadas las dos terminologías. 
e informaciones asociadas a la salud. En este contexto, el derecho a la salud consiste en el derecho a instalaciones, bienes, condiciones y servicios necesarios para alcanzar el más alto nivel posible de salud. Por tanto, el entendimiento de este derecho no se limita a los cuidados en salud, sino que involucra el derecho a los determinantes sociales que impactan en el proceso salud-enfermedad (United Nations Economic and Social Council, 2000).

\section{Determinantes sociales de la salud}

Conforme estas afirmaciones, es imprescindible revisar el concepto de determinantes sociales de la salud. Estos son comprendidos como las condiciones en las cuales las personas nacen, crecen, trabajan, viven y se envejecen, y que originan la mayor carga de enfermedad (World Health Organization, 2010). Su surgimiento se contextualiza en la siguiente dinámica: mecanismos políticos, económicos y sociales, como el mercado de trabajo, el sistema educativo, las instituciones políticas, valores sociales y culturales, crean y reproducen una serie de posiciones socioeconómicas, que conducen a la estratificación de las poblaciones, en consonancia con su nivel de ingresos, educación, ocupación, genero, raza/etnia y otros factores.

Los determinantes sociales son clasificados en dos categorías, estructurales e intermediarios. Los determinantes estructurales son aquellos que producen divisiones de clases en la sociedad y que definen posiciones socioeconómicas individuales, encuadradas en las jerarquías de poder, acceso a los recursos y prestigio. este tipo de determinantes es considerado el más relevante, en razón de causar estratificación de la sociedad y en consecuencia, inequidades en salud. Ejemplos de determinantes sociales estructurales son la distribución de la renta y la discriminación (por motivo de raza, género u orientación sexual) (World Health Organization, 2010).

Los determinantes sociales intermediarios son circunstancias materiales y circunstancias psicosociales; por ejemplo, el estrés, las relaciones interpersonales y el apoyo social. Nutrición, actividad física, consumo de tabaco, alcohol y factores genéticos también son considerados en este grupo (World Health Organization, 2010).

\section{Elementos del derecho a la salud}

Este derecho contiene "elementos interrelacionados y esenciales, que se manifiestan en todas sus formas y niveles" (United Nations Economic and Social Council, 2000, p. 4). Dichos elementos han sido formulados con el propósito de otorgar una mayor precisión al contenido de este derecho, y a continuación son explicados.

La disponibilidad es entendida como el número suficiente de bienes, instalaciones y servicios de salud; implica número suficiente de profesionales de salud (United Nations Economic and Social Council, 2000). 
La accesibilidad consiste en que las instalaciones, bienes y servicios de salud sean accesibles a todas las personas sin discriminación alguna. Este elemento debe ser alcanzado en las siguientes dimensiones: no discriminación, es decir, los grupos vulnerables de la población, deben tener garantizada la accesibilidad, de derecho y de hecho; la accesibilidad física, engloba la construcción de instalaciones de salud que faciliten la movilización de las personas con discapacidad física; la accesibilidad económica, que envuelve la asequibilidad de los cuidados en salud y de sus determinantes sociales subyacentes, y por último, la accesibilidad a las informaciones, que abarca el derecho de buscar, recibir y divulgar informaciones relacionadas con temas de salud (United Nations Economic and Social Council, 2000).

La aceptabilidad es el elemento relacionado directamente con la ética; como es establecido en su definición, los servicios de salud deben respetar la ética médica, ser culturalmente apropiados y respetar las especificidades culturales. Asimismo, instalaciones, bienes y servicios deben considerar la perspectiva de género y los requerimientos de cada ciclo de vida y, además, conservar el respeto por el principio de la confidencialidad (United Nations Economic and Social Council, 2000).

La calidad consiste en la adecuación de las instalaciones, bienes y servicios desde el punto de vista científico y médico; para lo anterior es necesaria la capacitación adecuada de los profesionales de la salud, suministro de medicamentos científicamente aprobados, equipos hospitalarios adecuados y condiciones sanitarias apropiadas y seguras (United Nations Economic and Social Council, 2000).

\section{Obligaciones estatales}

La Observación 14, enfatiza obligaciones, generales, especificas, internacionales y esenciales; estas son explicadas a continuación.

Las obligaciones generales engloban la realización progresiva del derecho a la salud; quiere decir que la legislación de los derechos humanos reconoce el hecho de que la realización inmediata de este derecho no siempre es posible. Sin embargo, los Estados deben presentar un plan a largo plazo que establezca pasos concretos, compromisos políticos y medidas financieras con el objeto de su materialización (United Nations Economic and Social Council, 2000).

Con respecto a las obligaciones específicas, el derecho a la salud, así como todos los derechos humanos, impone tres tipos de deberes: respetar, proteger y cumplir. Respetar, implica la obligación negativa de no interferir directa o indirectamente con el disfrute del derecho a la salud; por ejemplo, evitar la imposición de restricciones a la accesibilidad de las instalaciones, bienes y servicios. Del mismo modo, los Estados deben abstenerse de imponer prácticas, tratamientos y políticas discriminatorias relacionadas con la salud de grupos vulnerables, así como evitar retener informaciones sobre 
salud (United Nations Economic and Social Council, 2000).

La obligación de proteger exige la adopción de mecanismos que eviten que terceros o instancias privadas violen el derecho a la salud; luego, es imperativa la promulgación de leyes que supervisen la privatización de los servicios de salud, con el propósito de que sean accesibles, aceptables, disponibles y de calidad (United Nations Economic and Social Council, 2000).

El deber de cumplir se refiere a la promulgación de normativas y medidas orientadas a la satisfacción del derecho a la salud; por ejemplo, el cumplimiento se manifiesta en la adopción de políticas nacionales, que garanticen la provisión de los cuidados en salud y el acceso equitativo a sus determinantes (United Nations Economic and Social Council, 2000).

Considerando estos fundamentos, las obligaciones específicas atribuyen a los Estados el deber de reconocer la interdependencia del derecho a la salud con otros derechos humanos; con base en esta característica, respetar, proteger y cumplir el derecho a la salud llevan a la disminución del impacto de la vulnerabilidad de determinados grupos.

En relación con las obligaciones internacionales, la Observación 14 establece que el respeto al derecho a la salud incorpora la cooperación internacional, es decir, se demarca la responsabilidad de los Estados parte sobre la asistencia humanitaria, incluyendo ayuda a personas refugiadas y víctimas del desplazamiento interno; además, la cooperación internacional también abarca la adopción de medidas preventivas para evitar que terceros violen este derecho en otros países (United Nations Economic and Social Council, 2000).

Las obligaciones esenciales son comprendidas como "el contenido tangible de la dignidad humana" (Albuquerque, 2009 , p. 97), en razón de que la no satisfacción de los bienes contenidos en estos deberes es un obstáculo para la vida digna.

Estas obligaciones son seis. Primero, garantizar del derecho al acceso a bienes, instalaciones y servicios de salud sin discriminación; segundo, asegurar el acceso a nutrición adecuada y segura; tercero, garantizar el acceso a habitación, condiciones sanitarias y agua potable; cuarto, suministrar medicamentos esenciales, de acuerdo con la oms; quinto, fiscalizar la distribución equitativa de bienes, instalaciones y servicios de salud, y finalmente aplicar un plan de acción pública, consonantes con estudios epidemiológicos que faciliten el monitoreo de los resultados obtenidos (United Nations Economic and Social Council, 2000).

A continuación, serán presentadas las nociones teóricas del derecho a la salud bucal.

\section{Derecho a la salud bucal}

Para abordar este derecho es imprescindible delimitar el concepto de salud 
bucal. El Informe del cirujano general es un documento que marcó la inclusión de la salud buco dental, como componente de la salud general y como determinante de la calidad de vida (National Institute of Dental and Craniofacial Research, 2000).

La evidencia científica apoya la relación entre la salud bucal y general, justificada en los factores de riesgo compartidos entre las patologías como periodontitis ${ }^{4}$ con la diabetes, condiciones cardiacas y enfermedades pulmonares obstructivas (Persson, 2008; Chappel y Genco, 2013). Uno de estos factores de riesgo es la malnutrición, ${ }^{5}$ que influye, en el desarrollo de periodontitis, defectos del esmalte ${ }^{6} \mathrm{y}$ en la progresión de la caries. ${ }^{7}$ Otro factor

$4 \quad$ Enfermedad inflamatoria de los tejidos de soporte de los dientes, causada por microrganismos específicos y que resulta en la destrucción progresiva del ligamento periodontal y del hueso alveolar, con formación de bolsa, retracción gingival, o ambas (Lindhe y Niklaus, 2015).

5 Existen dos tipos de mal nutrición: el primero está asociado a las deficiencias nutricionales y el segundo, a los excesos dietarios, que llevan a enfermedades como la obesidad, diabetes, enfermedades cardiovasculares y enfermedades bucodentales. En este artículo son considerados estos dos tipos de mal nutrición (Petersen, World Health Organization, 2003).

$6 \quad$ Tejido inerte, duro y acelular que cubre la corona anatómica de los dientes (Infante, 2009).

7 Enfermedad crónica que aparece como resultado de la disolución mineral de los tejidos dentales, consecuencia de los ácidos producidos por las bacterias, las cuales metabolizan carbohidratos, particularmente sacarosa proveniente de la dieta (Infante, 2009). de riesgo es el tabaquismo, que induce la aparición de cáncer bucal, periodontitis y defectos faciales congénitos. Por consiguiente, las enfermedades bucales han sido relacionadas con los estilos de vida que generan las enfermedades crónicas y han sido calificadas como problemas de salud pública (Ramírez y Contreras, 2007).

La literatura odontológica refiere que la periodontitis no se limita a las encías y al hueso alveolar, ya que esta enfermedad induce una respuesta inflamatoria sistémica, evidente en los niveles de proteína $C$ reactiva y fibrinógeno, sustancias que aumentan en el suero sanguíneo, en respuesta a la inflamación y, además, son consideradas factores de riesgo para infarto agudo de miocardio, accidente cerebrovascular y enfermedad vascular periférica (Persson, 2008). Además de ser un factor de riesgo para la aparición de este tipo de alteraciones, la periodontitis no tratada está relacionada con el frágil control de la glicemia, nefropatía diabética, aumento de la mortalidad de los pacientes diabéticos y desarrollo de trastornos del embarazo, como preclamsia, bajo peso al nacer y parto prematuro (Da Silva et al., 2012).

Por otro lado, la perspectiva de la salud bucal como componente de la salud general, en los instrumentos normativos internacionales, ha sido incentivada por la oms, agencia que ha promulgado directrices que incluyen las Resoluciones de la Asamblea Mundial de la Salud, particularmente la Resolución 36.14 de 1983 y la Resolución 42.39 de 1989 
(oms, Asamblea Mundial de la Salud, 2007). Igualmente, a través del Programa de Salud Bucal Global, se determina la necesidad de capacitar a los agentes públicos de los Estados, con la meta de incluir la promoción de la salud bucal en los programas de promoción de la salud general (Petersen, 2014).

Aunque la oms establece una visión ampliada de la salud bucal, ha sido afirmado que su definición contiene ambigüedades, especialmente en el ámbito de la investigación odontológica; es decir, en este campo, predomina la perspectiva de la boca como una estructura anatómica aislada, que puede o no estar saludable, omitiendo a la persona como un todo y sus contextos. Siendo así, los abordajes en investigación odontológica no siempre garantizan la relación entre salud bucal y salud general, aclamada por la oms (Locker, 1997).

Con base en estos fundamentos, aproximarnos al significado de la salud bucal implica que el enfoque no es la boca en sí misma, sino el individuo y las vías en que las enfermedades confinadas a esta estructura, amenazan la salud, el bienestar y la calidad de vida. Así, la definición de la salud bucal está integrada al concepto de la salud general, en la medida en que las enfermedades buco dentales no difieren de las alteraciones en otros órganos. De este modo, disfrutar de una salud bucal adecuada se deriva en el respeto a la dignidad humana, marco axiológico compartido entre los derechos humanos y la bioética.
Es posible inferir que la salud bucal, de modo similar a la salud general no solo está asociada a los cuidados, sino también está intrínsecamente vinculada a los determinantes sociales, a los elementos del derecho a la salud y, además, requiere el cumplimiento de las obligaciones estatales, explicados detalladamente en la Observación 14 (United Nations Economic and Social Council, 2000).

Importa señalar qué instrumentos normativos han dado visibilidad a este derecho. Destáquese el Reporte de la Salud Bucal Mundial (World Dental Federation, 2015); igualmente, la Carta de Brasilia sobre salud bucal en las Américas (Encuentro Latinoamericano de Coordinadores Nacionales de Salud Bucal, 2009) enfatiza este derecho, en razón de que realza las responsabilidades estatales en el campo de la salud bucal.

Sin embargo, es notoria la falta de contenido concreto del derecho a la salud bucal; es decir, sus fundamentos teóricos no han sido desarrollados, a diferencia del derecho a la salud, cuyo contenido fue explícito en la Observación 14 (United Nations Economic and Social Council, 2000).

La ausencia de un contenido explícito del derecho a la salud bucal puede potencialmente perjudicar la participación de individuos y comunidades, limitando su rol de sujetos activos en este campo de la salud. Esta afirmación se basa en datos de una encuesta realizada en Brasil, solicitada por el Consejo Federal 
de Odontología (2016), en la que se demostró que una parte significativa de este país desconoce su derecho a la salud bucal. En este orden de ideas, Narvai (2006) se refiere al carácter ético-político de los sujetos, como elemento del Sistema Único de Salud, ${ }^{8}$ en el ámbito de la salud bucodental.

La búsqueda por el contenido teórico del derecho a la salud bucal lleva a abordar nociones sobre la salud bucal colectiva, ${ }^{9}$ que defiende la garantía al acceso a los recursos necesarios para que los cuidados odontológicos sean efectivamente un derecho humano.

Considerando estos fundamentos teóricos, a continuación, se presenta la segunda parte de este manuscrito.

\section{Metodología}

Este es un estudio cualitativo, exploratorio de base empírica y documental, realizado durante el primer semestre de 2016. El propósito de este análisis es determinar si existe o no interface

8 Reconocido como uno de los mayores sistemas públicos de salud del mundo, instituido en 1988, por la Constitución Federal Brasilera, fundamentado en la universalidad, integralidad, participación social y equidad (Portal da Saúde, 2012b).

9 Movimiento teórico-político originado a partir de la Reforma Sanitaria Brasilera; tiene la pretensión de generar una ruptura epistemológica con la odontología y rechaza la odontología del mercado, cuya práctica se centra en la asistencia odontológica al individuo enfermo y realizada por un sujeto individual (Narvai, 2006). entre el referencial teórico-normativo del derecho a la salud y las acepciones del Reporte Mundial de Salud Bucal de la oms (Petersen, 2003). Para el alcance de este objetivo, fue utilizada la metodología de análisis de prácticas discursivas propuesta por Spink y Giménez (1994), cuyo enfoque es el lenguaje utilizado, en este caso concreto, aplicado a un documento de dominio público. Así, las prácticas discursivas consisten en "las formas por las cuales las personas, por medio del lenguaje, producen sentidos y se posicionan en las relaciones sociales cotidianas" (Spink, 2010, p. 27). Los elementos de las prácticas discursivas son la dinámica, es decir, enunciados orientados por voces, las formas o speech genres ${ }^{10}$ y los repertorios lingüísticos.

Considerando el objetivo de esta investigación (determinar si el Reporte Mundial de Salud Bucal de la oms se constituye en una herramienta teórico-política para alcanzar la realización del derecho a la salud bucal), importa enfocar los repertorios lingüísticos, definidos como "términos, conceptos, lugares comunes y figuras del lenguaje que determinan posibilidades de construcción de sentidos" (Spink, 2010, p. 32). Los repertorios son divulgados de formas diversas y no son aprendidos formalmente; ejemplo de repertorios lingüísticos son los libros, revistas, películas, imágenes y expresiones artísticas.

10 Géneros de habla; esta expresión se refiere a las formas estables del habla que posibilitan la comunicación y son influenciadas por la expresividad propia de la cultura. 
La selección de esta metodología de análisis se justifica en que el Reporte Mundial de Salud Bucal de la oms, documento para ser analizado, consiste en prácticas discursivas producidas por una autoridad internacional en salud bucal, dirigida a los Estados parte. Siendo así, la oms11 se constituye en el órgano internacional que tiene como meta alcanzar un futuro más saludable para todas las personas; esta autoridad trabaja en asociación con los Gobiernos y otros socios, con el fin de asegurar el más alto nivel posible de salud para todos (World Health Organization, 2016). El examen de sus repertorios lingüísticos revelará la postura de esta agencia de las Naciones Unidas sobre el derecho a la salud bucal y la incorporación de las afirmaciones del referencial teórico-normativo del derecho a la salud en el documento mencionado.

A continuación, serán explicados los pasos ejecutados en el examen. Primero, fue realizada la lectura fluctuante $e^{12} \mathrm{y}$ breve presentación de su contenido;

11 Caracterizada como instancia de producción bioética integrada al Sistema das Naciones Unidas, que cuenta con oficinas específicas con competencia en esta área del conocimiento. Esta denominación es basada en la perspectiva de la bioética institucional, propuesta por Albuquerque (2010), que tiene como objeto el estudio de los órganos de la ética ligados a la medicina, ciencias de la vida y tecnologías asociadas, su formación y actividades.

12 Consiste en la primera etapa del proceso de análisis de material empírico en la pesquisa cualitativa; equivale a lectura en profundidad, la cual orienta al dominio del contenido del texto (Crevelim y Peduzzi, 2005). segundo, la elaboración de mapas de asociación de ideas, que consisten en instrumentos de visualización del proceso de análisis de las prácticas discursivas, que permiten la identificación de los repertorios lingüísticos (Spink, 2010) y que, consiguientemente, inducen a la creación de categorías relacionadas con el marco referencial adoptado en este estudio; es decir, el derecho a la salud, conforme a la Observación 14 de 2000. Por tanto, dicha categorización obedeció a la siguiente tripartición: contenido del derecho humano a la salud, que incluye sus determinantes sociales, elementos del derecho humano a la salud y las obligaciones estatales. El tercer paso consistió en la elaboración de interpretaciones sobre estos mapas.

Después de la explicación de los pasos metodológicos empleados, se inicia la presentación del contenido del Reporte Mundial de Salud Bucal.

\section{Documento objeto de análisis}

El Programa de Salud Bucal Global de la oms es un programa técnico componente del Departamento de Prevención de Enfermedades no Trasmisibles y Promoción de la Salud, que estimula el desarrollo de políticas globales en promoción de la salud bucal y prevención de las dolencias bucodentales. En el marco de este programa, fue publicado en 2003 el Reporte Mundial de Salud Bucal, documento que formula la política y las estrategias necesarias para alcanzar el mejoramiento de la salud bucal global en el siglo xxi (Petersen, 2003). 
Cabe recordar que la realización del derecho humano a la salud puede ser alcanzada por medio de diversas acciones; así, la implementación de programas de salud por parte de la oms es una de estas acciones (United Nations Economic and Social Council, 2000). Por lo tanto, el presente análisis buscará verificar si el documento en examen es una herramienta política-teórica que facilite la satisfacción de este derecho.

El Reporte Mundial de Salud Bucal se divide en cuatro partes. La primera presenta las bases políticas del Programa de Salud Bucal; la segunda desarrolla su marco político; en la tercera, son referidas estrategias y abordajes para prevención y promoción de la salud bucal, y en la cuarta parte se establecen las áreas prioritarias de acción para la salud bucal global. En el presente artículo, el Reporte Mundial de Salud Bucal será presentado en dos grandes partes: en una son integradas las bases y el marco político, en razón de que exponen cuestiones similares, por ejemplo, la salud bucal como componente de la salud general, la reducción de la carga de las enfermedades bucales en el mundo y los factores de riesgo para estas dolencias; estos puntos facilitan su incorporación en un solo ítem. En la otra parte serán tratadas las nociones de la promoción de la salud bucal y las alusiones a las áreas prioritarias de acción serán presentadas a lo largo de estas dos secciones.

\section{Bases y marco político del Programa de Salud Bucal Global}

La primera parte del reporte enfatiza que la salud bucal es parte esencial de la salud general y factor determinante para la calidad de vida. Adicionalmente, son referidas acepciones sobre la carga de las enfermedades bucales, explicando en detalle las condiciones más prevalentes y su distribución en el mundo. De esta manera, establece que todas las enfermedades de la boca son consideradas como problemas de salud pública, por cuenta de su amplia extensión en el mundo y su impacto en la reducción de la calidad de vida (Petersen, 2003).

En este punto son referidas algunas patologías como la caries y la enfermedad periodontal, consideradas históricamente como las dos entidades que causan mayor carga en salud bucodental a nivel global; además, el cáncer faríngeo y oral son reconocidos como enfermedades que imponen desafíos para los programas de salud bucal. Por otro lado, son abordados el trauma oro-dental y las mal oclusiones, ${ }^{13}$ que a pesar de no ser categorizadas como enfermedades bucales, sí se piensan como alteraciones dentales que afectan la calidad de viday cuyo tratamiento es justificado con el propósito de mejorar el bienestar social y psicológico de la persona (Petersen, 2003).

13 Representan desvíos de la normalidad de los arcos dentarios, del esqueleto facial o de ambos, que se reflejan en las funciones del sistema estomatognático, en la apariencia física y autoestima de los individuos afectados (Suliano et al., 2007). 
Otro desafío para la salud buco dental es el tratamiento de las anomalías cráneo faciales, como labio y paladar fisurado, ${ }^{14}$ esta alteración está asociada a causas como el tabaquismo materno, consumo de alcohol y deficiencias nutricionales. El reporte resalta otras enfermedades que exigen cuidados especiales en salud bucal, por ejemplo, síndrome de Down, parálisis cerebral, deficiencias intelectuales, trastornos del desarrollo y desordenes hereditarios/genéticos que provocan defectos orofaciales (Petersen, 2003).

En este sentido, gestores y tomadores de decisión en salud requieren herramientas para monitorear las necesidades de la población, crear estrategias de intervención e implementar políticas que faciliten el mejoramiento de los sistemas de salud bucal. Por tanto, la oms (2003) estimula a los Estados miembros para que establezcan sistemas de información bucodental, que incorporen mecanismos de vigilancia epidemiológica, cobertura de servicios para la población, reportes administrativos y manejo de recursos, calidad de los cuidados y evaluación de los resultados. De este modo, los sistemas

14 Reconocida científicamente como fisura labio palatina y considerada como una de las anomalías congénitas orofaciales más frecuentes. Esta malformación compromete el tercio medio de la cara y es ocasionada por la falta de fusión de los huesos maxilares, durante la sexta y décima semana de vida intrauterina. Puede ser un hallazgo aislado o presentarse en asociación con otros disturbios, como componente de un síndrome (Cymrot et al., 2010). de información bucal son considerados en las áreas prioritarias en salud bucal global en el reporte en análisis (Petersen, 2003).

El Reporte Mundial de Salud Bucal determina que la mayor carga de las enfermedades que afectan la cavidad oral es experimentada por los grupos marginalizados social y económicamente; por tanto, el abordaje fundamentado en los factores de riesgo tiene como núcleo el mejoramiento de las condiciones de salud para toda la población, con el fin de reducir las inequidades en este campo de la salud.

El reporte en mención establece cuatro grandes metas que constituyen el marco político del Programa de Salud Bucal, las cuales consisten en: reducir la carga de las enfermedades bucales y la incapacidad, especialmente en las poblaciones marginalizadas; demarcar la promoción de estilos de vida saludables y la reducción de factores de riesgo para la salud bucal, originados a partir de factores sociales, económicos, ambientales y comportamentales; desarrollar sistemas de salud bucal equitativos, que mejoren los resultados en este campo, que respondan a las demandas de las personas y que sean financieramente justos, y crear políticas de salud bucal, fundamentadas en su inclusión en los programas nacionales de salud (Petersen, 2003). A continuación, es presentada la segunda parte del Reporte Mundial de Salud Bucal. 
Estrategias y abordajes en prevención de la enfermedad y promoción de la salud

El documento refuerza la idea de que la promoción de la salud va más allá de los cuidados; en este contexto, disponer de una vida saludable es el mayor recurso para el desarrollo social, personal y económico; así, las acciones en promoción de la salud deben tener como objeto el hecho de que determinantes políticos, económicos, sociales y culturales tengan como núcleo la calidad de vida (Petersen, 2003).

Cabe recordar que las condiciones precarias de residencia, bajo nivel educativo y ausencia de tradiciones y creencias en torno a la salud bucal influyen en el surgimiento de las enfermedades. En el documento mencionado, estos factores son reconocidos como determinantes socioculturales; por consiguiente, la promoción de la salud bucal a través de las escuelas y colegios es contemplada como otra área prioritaria de acción, entendiendo la carga significativa de las enfermedades bucales en los niños y adolescentes (Petersen, 2003).

En este orden de ideas, el reporte establece que el enfoque ampliado de la promoción de la salud abarca los determinantes sociales de esta con el objeto de reducir riesgos, a través de políticas y acciones. Este documento reconoce que la fuerte influencia del bajo nivel socioeconómico en las desigualdades en salud bucal entre regiones y países está relacionada con la raza, etnia, edad, el género y estado general de salud. En este sentido, afirma que la persistencia de grupos desatendidos es un hecho social que manifiesta situaciones de discriminación y estigmatización en el campo de la salud bucal. El reporte determina que la reducción de las desigualdades requiere abordajes de amplio alcance y que incluyan la optimización del acceso a los cuidados en salud (Petersen, 2003).

\section{Resultados y discusión}

En esta sección serán presentados los mapas de asociación de ideas, repertorios lingüísticos e inferencias relacionadas con la interface entre el derecho a la salud bucal y el derecho a la salud, demarcadas en el Reporte Mundial de la Salud Bucal.

\section{1) Ausencia de referencias al derecho a la salud bucal.}

El primer sentido por ser discutido es la completa omisión de referencias al derecho a la salud o al derecho a la salud bucal, en el marco político y áreas prioritarias de acción expuestas en el reporte; importa transcribir las definiciones de salud bucal presentadas en este documento.

La salud bucal es integral y esencial a la salud general. La salud bucal es más que tener dientes en buen estado; ella es integral a la salud general y esencial para el bienestar. Esto implica estar libre de dolor crónico oro facial, cáncer bucal y faríngeo, 
lesiones de los tejidos, defectos de nacimiento y otras alteraciones que afectan el complejo cráneo-facial. (Petersen, 2003, p. 3)

La noción de la salud bucal como determinante de la calidad de vida se resalta en el siguiente enunciado:

El complejo cráneo facial nos permite hablar, sonreír, besar, tocar, masticar y deglutir. Proporciona protección contra infecciones microbianas y amenazas ambientales. Las enfermedades bucales restringen las actividades en los estudios, trabajo y hogar, causando millones de horas perdidas por año en el mundo. Además, el impacto psicosocial de estas enfermedades, reduce significativamente la calidad de vida. (Petersen, 2003, p. 3)

Obsérvese que en las dos conceptuaciones se tiene en cuenta la amplitud del espectro de la salud bucal, al destacar que es esencial para la salud general y que se constituye en un factor determinante para la calidad de vida; no obstante, la amplitud de este concepto no eleva la salud bucal a la categoría de derecho humano a ser garantizado por el Estado.

Con respecto a la salud bucal como determinante para la calidad de vida, se afirma concretamente la funcionalidad del complejo cráneo facial, las limitaciones que imponen las enfermedades de la boca y su impacto psicosocial; sin embargo, esta comprensión se revela insuficiente, en la medida en que la salud de la boca, como determinante de la calidad de vida, conduce a vivir una vida digna; siendo así, ella se constituye en un bien ético que debe ser protegido y garantizado por el Estado. Por lo tanto, se interpreta que el concepto de salud bucal proferido en el reporte se limita a los cuidados en salud buco dental y se opone al espíritu ampliado que pretende plasmar en sus bases políticas.

Adicionalmente, desde la perspectiva del referencial del derecho a la salud, la significación de la salud bucal expuesta demuestra un vacío relacionado con los determinantes sociales, en razón de que, al expresar la esencialidad de la salud bucal, se manifiesta únicamente en la esfera clínica, y de esta manera se omite el amplio número de determinantes que condicionan la aparición y desarrollo de las patologías bucodentales. A pesar de que la alusión a los determinantes sociales de la salud bucal fue demarcada a lo largo del contenido del reporte, estos no fueron considerados en las definiciones expuestas.

Además, hubo ausencia de acepciones sobre el disfrute de las instalaciones, bienes y servicios de salud odontológicos. Es necesario aclarar que en este campo de la salud, las instalaciones y bienes involucran no solamente los consultorios u hospitales donde se presta el servicio, sino también la silla odontológica, instrumental, aparatos de rayos $\mathrm{X}$, autoclaves, así como diversidad de 
biomateriales ${ }^{15}$ imprescindibles para la atención clínica.

\section{2) Obligaciones estatales en salud bucal}

Las áreas prioritarias de acción señalan tópicos como el flúor y la nutrición, temas que se entrelazan con las obligaciones esenciales prescritas en la Observación 14, que enfatiza el deber de asegurar acceso al agua potable y segura, así como garantizar la alimentación adecuada. Sin embargo, en el reporte el acceso al agua fluorizada y a la nutrición esencial no se categorizan como derechos humanos, ni como un deber estatal; simplemente se explica su influencia en la salud bucal y establecen desafíos globales para cada área.

Un fragmento relacionado con la salud bucal y el flúor indica lo siguiente: "El flúor ha sido utilizado a escala global con muchos beneficios [...] Sin embargo, poblaciones en países en vía de desarrollo no tienen acceso al flúor para la prevención de la caries [...]" (Petersen, 2003, p. 19).

El flúor es el mecanismo más efectivo para la prevención de la caries dental, por tanto, la exposición permanente al flúor resulta en la reducción de sus

15 Sustancia o combinación de sustancias, naturales o no, que se integran armónicamente con los sistemas biológicos, sin causarles daño alguno y sin ser rechazados; su objeto es tratar, aumentar o sustituir tejidos, órganos o funciones (Villegas, Naranjo y Gómez, 2008). niveles. No obstante, también se reconoce que no es posible alcanzar un nivel de caries sin que se presente algún grado de fluorosis dental ${ }^{16}$ (Ramires y Buzalaf, 2007).

En Colombia, el Gobierno adoptó desde 1992 el programa de fluorización de la sal, como medida de prevención masiva de la caries (Galindo, 2016). eEste programa es considerado controvertido, por cuenta de la epidemia de fluorosis dental en niños, poco tiempo después de la adopción de esta política (Agudelo-Suárez et al., 2013; Ramírez et al., 2010; Universidad Nacional de Colombia, 2015). La fluorosis es piensa como un indicador tardío de intoxicación sistémica por flúor, que tiene lugar durante el periodo de desarrollo de los dientes; además, la ingestión sistémica del flúor alcanza huesos, riñones y neuronas (Galindo, 2016); por lo tanto, es imperativo un estricto control y vigilancia de la distribución del flúor en la población.

Ante la constatación de la forma indiscriminada de distribución de este elemento químico, en la sal y en el agua potable, se cuestiona la eticidad de esta política pública, particularmente en relación

16 Condición producida por el efecto tóxico del flúor, originada por la exposición del germen dental a este compuesto, durante su periodo de formación; consecuentemente aparecen defectos de mineralización del esmalte, caracterizados por manchas opacas o amarillas, que predisponen a la caries, sensibilidad dental y mal oclusiones (Cangussu et al., 2002). 
con el deber de vigilar su implementación. De este modo, se verifica que la afirmación expuesta en el Reporte Mundial de la Salud Bucal sobre la falta de programas de fluorización en los países en desarrollo no converge con la realidad experimentada en Colombia.

Además de estas inconsistencias, cabe aclarar que la obligación esencial de garantizar acceso a bienes, servicios e instalaciones de salud sin discriminación alguna no se invoca en el Reporte Mundial de la Salud Bucal. Esta ausencia es contraria a las metas sobre la reducción de la carga de las patologías bucodentales en las poblaciones marginalizadas, expuesta en dicho documento.

\section{Elementos del derecho a la salud bucal}

El reporte problematiza la persistencia de grupos que sufren alta carga de las enfermedades bucales; así, señala la prevalencia de dolencias bucales en África, Asia, Europa, América Latina y Estados Unidos. No obstante, la explicación de estos datos se interconecta solo con algunos elementos de este derecho (United Nations Economic and Social Council, 2000). Igualmente, no se demuestra la forma como la falta de satisfacción de estos elementos aumenta la carga de las patologías bucodentales.

Uno de los elementos mencionados en el reporte Mundial de la Salud Bucal es la accesibilidad; las limitaciones del acceso a los servicios de salud bucal apenas son reconocidas en el documento en examen y cuando se refiere a la pérdida de los dientes originada por la falta de tratamiento de la caries en los países de baja renta afirma: "En muchos países en desarrollo, el acceso a los servicios de salud bucal es limitado y los dientes no son tratados o son extraídos como consecuencia del dolor y la incomodidad" (Petersen, 2003, p. 4).

En el párrafo anterior puede verse que no son puntualizadas las dimensiones de la accesibilidad limitadas. cabe resaltar además que la omisión a la no discriminación como dimensión de la accesibilidad dificulta la comprensión sobre las dinámicas sociales y sanitarias que perjudican a los grupos vulnerables.

Otra dimensión de la accesibilidad omitida en el reporte es la accesibilidad física; esta dimensión no solo involucra el acceso geográfico a los servicios de salud y a sus determinantes sociales, sino que también implica la adaptación de las instalaciones odontológicas para facilitar los cuidados de las personas con deficiencias físicas. Esta última noción no fue referida en el documento analizado.

Una dimensión de la accesibilidad encontrada en el reporte es la accesibilidad económica:

Las enfermedades bucales son grandes problemas de salud pública. Su impacto en individuos y comunidades es alto [...] el tratamiento tradicional de estas dolencias es extremadamente costoso, constituyéndose en el cuarto tratamiento más oneroso en 
los países industrializados. (Petersen, 2003, p. 9)

Es decir, las patologías bucales por ser problemas de salud pública tienen impacto individual, en términos de tiempo de vida perdido, aumento de la morbilidad y costos para la sociedad. Este fragmento es convergente con una de las metas propuestas en el reporte, que demarca la necesidad de "Desarrollar sistemas de salud bucal que mejoren equitativamente los resultados en esta área, respondan a las demandas y legitimaciones de las personas y sean financieramente justos" (Petersen, 2003, p. 14).

A pesar de esta confluencia, este repertorio se refiere únicamente al pago por los servicios odontológicos, sin aludir a los costos derivados del disfrute de los determinantes sociales, influyentes en la salud de la boca. Por tanto, se constata nuevamente un enfoque limitado a los cuidados en salud bucal, que son opuestas a las definiciones sobre la salud bucal, expuestas al comienzo del reporte.

Es pertinente asentar que la disponibilidad es un condicionante de la accesibilidad y a su vez es influenciada por una secuencia de eventos, como la necesidad de atención médica, la demanda por el servicio y la oferta de los prestadores de salud. La demanda es dependiente de factores como los recursos económicos, costos de la atención, estado de salud percibido por la persona y gravedad percibida. En contrapartida, la necesidad se presenta en el momento cuando la persona tiene un problema de salud, independientemente de si considera que este problema requiere o no atención profesional (Ayala-García, 2014).

En consonancia con estas asertivas, la alusión a la disponibilidad y accesibilidad en el reporte se establecen en el siguiente fragmento.

El alto riesgo relativo de las enfermedades bucales se relaciona a determinantes socioculturales [...] Escaso acceso al agua potable o facilidades sanitarias son factores de riesgo ambientales para la salud bucal y para la salud general. El control de las enfermedades bucales también depende de la disponibilidad y accesibilidad al sistema de salud bucal, pero la reducción del riesgo sólo es posible si los servicios son orientados a los cuidados primarios y a la prevención. (Petersen, 2003, p. 15)

En este sentido, es notorio el reconocimiento sobre los determinantes sociales y su impacto en el desarrollo de las patologías bucales, afirmación que se coaduna con la noción inclusiva del derecho a la salud, expuesta en la Observación 14 y que confirma la intersección entre este derecho y el derecho a la salud bucal. Sin embargo, en este parágrafo se destaca que la reducción del riesgo a enfermarse solo es posible a través de los cuidados primarios y la prevención; de este modo, se constata reiterativamente el énfasis a los cuidados en salud bucal, sin considerar que los determinantes sociales aludidos también contribuyen a la disminución del riesgo. 
Otro aspecto relacionado con los elementos del derecho a la salud es la escasa mención de la aceptabilidad y la calidad. En el documento referenciado solo fue identificada una mención a la aceptabilidad, concretamente en el área de acción sobre las personas de la tercera edad, que refiere:

Es importante que los proveedores de servicios de salud reconozcan los factores psicosociales que afectan la salud y el bienestar de las personas de la tercera edad. Hay necesidad de proporcionar servicios de salud bucal que sean accesibles y aceptables para esta población. (Petersen, 2003, p. 26)

Conviene reforzar que la aceptabilidad implica que las instalaciones, bienes y servicios de salud deben ser respetuosos de las especificidades culturales de minorías e individuos, y además deben ser sensibles al género (United Nations Economic and Social Council, 2000). Con respecto a esta afirmación, la salud bucal de la mujer, poblaciones indígenas y personas con discapacidad no fue incluida dentro de las áreas prioritarias de acción propuestas en el reporte, considerando solamente a las personas de la tercera edad, niños, adolescentes y personas que padecen de hiv sida.

La ausencia de estas poblaciones es contradictoria, en la medida en que este documento reconoce las desigualdades en salud bucal ocasionadas por factores como la raza, el género y estado general de salud, crea poblaciones desatendidas.
En este sentido, una de las responsabilidades de la oms, establecidas en el reporte, es monitorear los patrones de las patologías bucales y analizar sus determinantes, enfatizando las poblaciones excluidas (Petersen, 2003).

La mujer es un agente multiplicador de las informaciones sobre promoción y prevención de las enfermedades bucodentales; este importante papel se interconecta con la garantía del derecho a la salud de la mujer y la lucha contra su discriminación (Organización de las Naciones Unidas [onu], 1979). Además de su rol social, cabe recordar que la mujer embarazada sufre cambios fisiológicos en su cavidad oral, particularmente en su periodonto, ocasionado por el desequilibrio hormonal; la encía presenta receptores de estrógeno y progesterona, lo que explica la respuesta gingival incrementada a la placa bacteriana (Lindhe y Niklaus, 2015). Estas razones justifican la inclusión de la mujer en las áreas prioritarias de acción de salud bucal global, con vistas a incentivar a los Estados para que adopten mecanismos que garanticen igualdad de condiciones para este grupo social.

En relación con los grupos indígenas, la concepción de la boca, prevención y tratamiento de las patologías, son determinados por la cultura, como es ponderado en la investigación etnográfica realizada en Colombia por Ospina-Lozano y Ortiz (2011), que relata el autoatendimiento odontológico en una comunidad indígena fundamentada en los saberes tradicionales con plantas. La 
pesquisa en mención demuestra que las comunidades indígenas no demandan los servicios de salud de forma frecuente y en las ocasiones cuando la atención odontológica es prestada por los hospitales locales, la atención se da "en un escenario estresante y poco apropiado para la situación intercultural" (Ospina-Lozano y Ortiz, 2011, p. 91).

En lo relativo a la persona con discapacidad, está bien establecido en la literatura científica su mayor riesgo de desarrollar caries y enfermedad periodontal, por cuenta de factores que favorecen el acumulo de placa bacteriana, por ejemplo, el grado de limitación física o mental, su inadecuada nutrición o la negligencia de sus cuidadores en relación con su higiene bucal. (Patrick et al. , 2010). Esta lamentable situación trae como consecuencia la ejecución de tratamientos odontológicos mutiladores, realizados en muchas ocasiones sin el consentimiento informado de estas personas (Queiroz et al., 2014). Por lo tanto, es urgente la adopción de programas y políticas en salud bucal dirigidos a esta población, las cuales deben ser conjugadas con la capacitación de los profesionales de la odontología, para que les sea brindado un servicio fundamentado en los preceptos del derecho a la salud y de la bioética.

Con respecto al elemento de la calidad, fue verificado que en el reporte solo es mencionado una vez, específicamente en el área de acción sobre los sistemas de salud, que afirma que "En muchos países la capacidad y recursos [...] son aún insuficientes para asegurar disponibilidad y acceso a los servicios esenciales de salud de alta calidad [...] (Petersen, 2003, p. 27).

Obsérvese que la calidad de los servicios de salud bucal es considerada, pero el tema no es desarrollado en su totalidad, en el sentido de que el perfeccionamiento de la calidad de los servicios, bienes e instalaciones odontológicas no hace parte de las metas propuestas en el reporte. Para la oms, la calidad engloba los servicios y los sistemas de salud; por tanto, ellos deben orientar mecanismos para mejorar la calidad de los cuidados en seis dimensiones: efectividad, eficiencia, accesibilidad, centrados en el paciente, equidad y seguridad (World Health Organization, 2006).

\section{Conclusiones}

Las metas en salud bucal trazadas en el Reporte Mundial de Salud Bucal han sido consideradas un llamado a la consciencia a los actores gubernamentales sobre la relevancia de este campo de la salud; es decir, implican el deber ético de asegurar y proteger el derecho a la salud, incluyendo la salud de la boca. Siendo así, es imprescindible que las normas internacionales sobre el derecho a la salud sean el eje para la implementación global de programas en el campo odontológico.

Por otro lado, a pesar de que el contenido del Programa de Salud Bucal Global de la oms no presente naturaleza 
prescriptiva, su propósito es incentivar acciones locales en los países miembros, y así se constituye en una estrategia complementaria para alcanzar la satisfacción del derecho a la salud. Fue constatado que el Reporte Mundial de Salud Bucal no incorpora el enfoque basado en los derechos humanos, al desconsiderar acepciones al derecho a la salud bucal en sus conceptuaciones, objetivos y contenido. Al alejarse de este referencial, también se distancia de la bioética, en razón de que se desconsidera al paciente en sus múltiples contextos, que permean el proceso salud-enfermedad. Este hecho confluye con la frágil protección de grupos vulnerables y con el escaso aseguramiento de condiciones de vida dignas.

Los elementos del derecho a la salud establecidos en el documento enfatizan la accesibilidad y la disponibilidad, en detrimento de la aceptabilidad y la calidad; la escasa mención a la aceptabilidad converge con la ausencia de grupos en las áreas de acción prioritarias, como mujeres, poblaciones indígenas y personas con discapacidad. Este hecho es un asunto que toca a la bioética, ya que la salud bucal es un bien ético que debe ser protegido en igualdad de condiciones. La ausencia de estos grupos dificulta el enfrentamiento de determinantes sociales estructurales.

Para Hunt y Backman (2008), el derecho a la salud considera los resultados y procesos, es decir, la satisfacción de este derecho impone no solamente el acceso a los servicios, bienes e instala- ciones de salud, sino también la forma como se garantiza dicho acceso; es en este punto donde la aceptabilidad y la calidad deben ser destacadas.

En síntesis, el examen de los repertorios lingüísticos del Reporte Mundial de la Salud Bucal verificó que no se constituye en una herramienta política-teórica orientada a la satisfacción del derecho a la salud bucal, en la medida en que la perspectiva de este documento es limitada a los cuidados en salud bucal, no establece mecanismos para el efectivo enfrentamiento de los determinantes sociales y las obligaciones estatales de asegurar el derecho a la salud bucal, no son enfatizadas.

\section{Referencias}

Agudelo-Suárez, A., Martínez-Flórez, L., Madrid-Gutierrez, L.M., Vivares-Builes, A. y Rocha-Buelvas, A. (2013) Panorama de la fluorosis dental em Colombia. Una revisión exploratória de la literatura. Universitas Odontológica, 32(68),133145.

Albuquerque, A. (2010) Interface entre bioética e direitos humanos: perspectiva teórica, institucional e normativa (Tesis de Doctorado). Universidade de Brasília, Brasília, Brasil.

Albuquerque, A (2009). Direito à saúde: conteúdo, essencialidade e monitoramento. Revista CEJ, 48, 92-100.

Ayala-García, J. (2014). La salud en Colombia: más cobertura, pero menos acceso. Documentos de Trabajo sobre Economía Regional, 204,1-45. 
Cangussu, M.C.T, Narvai, P.C., CastellanosFernández, R., Djehizian, V. (2002). A fluorose dentária no Brasil: uma revisão crítica. Cad Saúde Pública,18 (1), 7-15.

Conselho Regional de Odontologia de Rio de Janeiro (2016). Pesquisa revela que brasileiros desconhecem ter direito a saúde bucal. Recuperado de http://www.cro-rj.org. br/pesquisa-revela-que-brasileiros-desconhecem-ter-direito-a-saude-bucal/

Chappel, I. y Genco, R. (2013). Diabetes and periodontal diseases: consensus report of the Joint EFP/AAP Workshop on Periodontitis and Systemic Diseases. Journal of Clinical Periodontoloy, 40(s14), s106-s112.

Crevelim, M. A. y Peduzz,i M. (2005). A participação da comunidade na equipe de saúde da família. Como estabelecer um projeto comum entre trabalhadores e usuários. Ciência e Saúde Coletiva, 10(2),323-331.

Cymrot, M., Sales, F., Teixeira, F., Teixeira, Jr F. et al. (2010). Prevalência dos tipos de fissuras em pacientes com fissuras labiopalatinas atendidos em um Hospital Pediátrico do Nordeste Brasileiro. Revista Brasileira de Cirugía Plástica, 25(4), 648-651.

Da Silva, G., Coutinho, S., Piscoya, M.D., Ximenes, R. y Jamelli, S. (2012). Periodontitis as a Risk Factor for Preeclampsia. Journal of Periodontology, 83(11), 1388-1396.

Encuentro Latinoamericano de Coordinadores de Nacionales de Salud Bucal. (2009). Carta de Brasilia sobre la salud bucal en las Américas. Recuperado de http://dab.saude.gov.br/docs/publicacoes/geral/carta_brasilia_salud_bucal_americas.pdf
Galindo, F. (2016) ¿Hasta cuándo seguiremos afectados por el flúor en la sal? Recuperado de http://www.elespectador.com/ opinion/hasta-cuando-seguiremos-afectados-el-fluor-sal

Hunt, P. y Backman, G. (2008). Health Systems and the Right to the Highest Attainable Standard of Health, 10(1), 81-92.

Infante, C. (2009). Desarrollo dental y estructuras de soporte. En Fundamentos para la evaluación del crecimiento, desarrollo y función craneofacial. En Fundamentos para la evaluación del crecimiento, desarrollo y función craneofacial (pp. 238-270). Bogotá: Universidad Nacional de Colombia.

Lindhe, J. y Niklaus, P. (2015). Clinical periodontology and implant dentistry. Oxford: Willey Blackwell.

Locker, D. (1997). Concepts of oral health, disease and the quality of life En D. Slade (Ed.), Measuring oral health and quality of life (pp. 11-23). Chapel Hill: University of North Carolina, Dental Ecology.

Narvai, P. (2006). Saúde bucal coletiva: caminhos da odontologia sanitária à bucalidade. Revista Saúde Coletiva, 40(No. especial), 141-147.

National Institute of Dental and Craniofacial Research (2000). Oral health in America: a report of the surgeon general. Recuperado de http://www.nidcr.nih. gov/DataStatistics/SurgeonGeneral/sgr/ chapl.htm\#whatis

Organização das Nações Unidas para a Educação, a Ciência e a Cultura, Unesco (2005). Declaração Universal sobre Bioética e Direitos Humanos. Cátedra 
Unesco de Bioética da Universidade de Brasília e Sociedade Brasileira de Bioética. Recuperado de http://bvsms.saude.gov. br/bvs/publicacoes/declaracao_univ_ bioetica_dir_hum.pdf

Organización Mundial de la Salud [oms] (2007). Salud bucodental: plan de acción para la promoción y la prevención integrada de la morbilidad. 60 Asamblea Mundial de la Salud. Recuperado de http://apps. who.int/iris/bitstream/10665/25842/1/ A60_R17-sp.pdf

Organización de las Naciones Unidas [ONU] (1979). Convención sobre la eliminación de todas las formas de discriminación contra la mujer. Recuperado de http:// www.iedf.org.mx/sites/DDHH/convenciones/10.pdf

Ospina-Lozano, E.J. y Ortíz, C.B. (2011). Autoatención en salud oral en el pueblo Yanacona de los Andes del sur de Colombia. Acta Odontológica Colombiana,1(2), 85-101.

Patrick, L., Anders, E. y Davis, L. (2010). Oral health of patients with intellectual disabilities: A systematic review. Special Care in Dentistry, 30(3), 110-117.

Persson, G. y Persson, R. (2008). Cardiovascular disease and periodontitis: an update on the associations and risk. Journal of Clinical Periodontology, 35(s8), 362-379.

Petersen, P. E. (2003). The World Oral Health Report. Recuperado de http://www. who.int/oral_health/media/en/orh_report03_en.pdf

Petersen, P. E. (2014). Strengthening of Oral Health Systems: Oral Health through Primary Health Care. Medical Principles and Practice, 23(1), 3-9.
Portal da Saúde Sistema Único de Saúde (2012a). Brasil sorridente. Recuperado de http://dab.saude.gov.br/portaldab/ ape_brasil_sorridente.php

Portal da Saúde Sistema Único de Saúde (2012b). Entenda o sus. Recuperado dehttp://portalsaude.saude.gov.br/index.php/cidadao/entenda-o-sus

Potter, V.R. y Potter, L. (1995). Global bioethics: converting sustainable development to global survival. Medicine and Global Survival, 2(3), 185-191.

Queiroz, F., Rodrigues, M.M., Cordeiro, J. G., Oliveira, A. et al. (2014). Avaliação das condições de saúde bucal dos portadores de necessidades especiais. Revista de Odontololia da UNESP, 43(6), 396-401.

Ramírez, J.H. y Contreras A. (2007) ¿Se debe considerar a la enfermedad periodontal un problema de salud pública en Colombia? Editorial Colombia Médica, 38(3),181-182.

Ramires, I. y Buzalaf, M. A. (2007). Fluoretação da agua de abastecimento público e seus benefícios no controle da cárie dentária, cinquenta anos no Brasil. Ciência E Saúde Coletiva, 12(4), 1057-1065.

Ramírez, B.S., Franco, A.M., Gómez, A.M. y Corrales, D.I. (2010). Fluorosis dental en escolares de instituciones educativas privadas. Revista Facultad de Odontololía Universidad de Antioquia, 21(2), 170-176.

Suliano, A.A., Rodríguez, M.J., Caldas, Jr. A., Fonte, P. y Carreiro, C (2007). Prevalência de maloclusão e sua associação com alterações funcionais do sistema estomatognático entre escolares. Cadernos de Saúde Pública, 23(8),1913-1923. 
Spink, M. J. y Giménes, M. G. (1994). Práticas discursivas e produção do sentido: apontamentos metodológicos para análises de discursos sobre a saúde e a doença. Saúde e Sociedade, 3(2),149-171.

Spink M.J. (2010). Linguagem e produção de sentidos no cotidiano. Rio de Janeiro: Centro Edelstein de Pesquisas Sociais.

Universidad Nacional de Colombia (2015). Colombia consume hasta seis veces más flúor del recomendado Recuperado de http://agenciadenoticias.unal.edu.co/ detalle/article/colombia-consume-hasta-seis-veces-mas-fluor-del-recomendado.html

United Nations Economic and Social Council (2000). The right to the highest attainable standard of health. Recuperado en http:// apps.who.int/disasters/repo/13849_files/o/UN_human_rights.htm

United Nations General Assembly (1966). International Covenant on Economic, Social and Cultural Rights. Recuperado de http://www.ohchr.org/Documents/ ProfessionalInterest/cescr.pdf
Villegas, A., Naranjo, E. y Gómez, D. (2008). Pruebas de biocompatibilidad de los materiales de uso odontológico: revisión de la literatura. Revista Estomatología, 16(2), 38-44.

World Health Organization (2010). A conceptual framework for action on the social determinants of heatlh. Social determinants of health discussion paper 2. Recuperado de http://www.who.int/sdhconference/ resources/ConceptualframeworkforactiononSDH_eng.pdf

World Health Organization (2016). About WHO. Recuperado en http://www.who. int/about/en/

World Dental Federation (2015). El desafio de las enfermedades buco dentales una llamada a la acción global. Recuperado en http:// www.fdiworldental.org/media/84768/ book_spreads_oh2_spanish.pdf

World Health Organization (2006). Quality of care, a process for making strategic choices in health systems. Recuperado en http:// www.who.int/management/quality/assurance/QualityCare_B.Def.pdf 